\title{
Food derived respiratory complex I inhibitors modify the effect of Leber hereditary optic neuropathy mutations
}

\section{Ester L}

esterlop@unizar.es

\section{Sonia Emperador ${ }^{\mathrm{a}, \mathrm{b}, \mathrm{c}}$}

seortiz@unizar.es

Carmen Hernández-Ainsa ${ }^{\mathrm{a}, \mathrm{b}}$

carmenha@unizar.es

Julio Montoyaa, b, c

jmontoya@unizar.es

\section{Pilar Bayona-Bafaluy ${ }^{\mathrm{a}, \mathrm{b}, \mathrm{c}, * *}$}

pbayona@unizar.es

Eduardo Ruiz-Pesini ${ }^{a, b, c, ~ d, ~ * ~}$

eduruiz@unizar.es

a Departamento de Bioquímica, Biología Molecular y Celular. Universidad de Zaragoza, Zaragoza, Spain

bInstituto de Investigación Sanitaria de Aragón (IIS Aragón), Zaragoza, Spain

${ }^{\mathbf{c}}$ Centro de Investigaciones Biomédicas En Red de Enfermedades Raras (CIBERER), Zaragoza, Spain

dFundación ARAID, Zaragoza, Spain

${ }^{*}$ Corresponding author. Departamento de Bioquímica, Biología Molecular y Celular. Universidad de Zaragoza, C/ Miguel Servet, 177, 50013, Zaragoza, Spain.

${ }^{* *}$ Corresponding author. Departamento de Bioquímica, Biología Molecular y Celular. Universidad de Zaragoza, C/ Miguel Servet, 177, 50013, Zaragoza, Spain.

\section{Abstract}

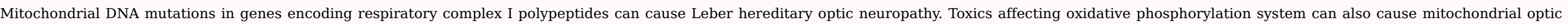

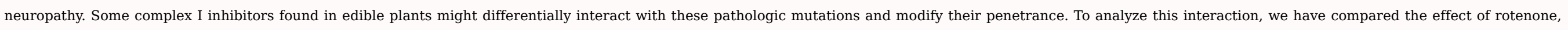

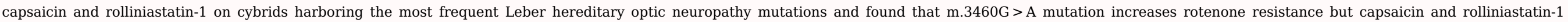
susceptibility. Thus, to explain the pathogenicity of mitochondrial diseases due to mitochondrial DNA mutations, their potential interactions with environment factors will have to be considered.

Keywords: Respiratory complex I; Xenobiotic; Mitochondrial DNA; Leber hereditary optic neuropathy; Gene-environment interaction

\section{Introduction}

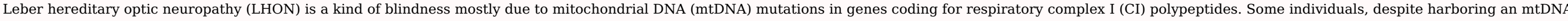

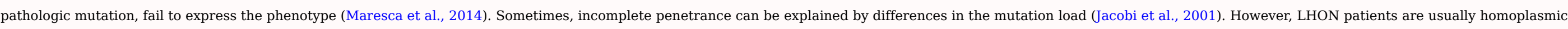

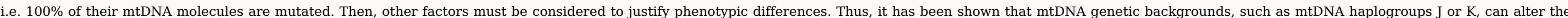




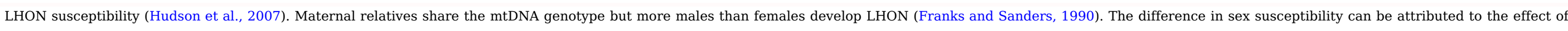

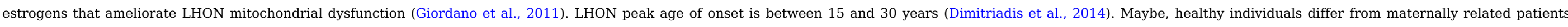
because they have not reached a particular age. However, reports of healthy individuals, older than patients, are not rare in LHON homoplasmic mutant pedigrees (Vilkki et al., 1989).

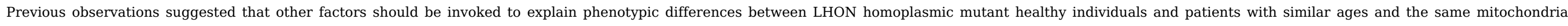

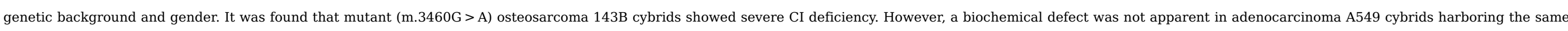

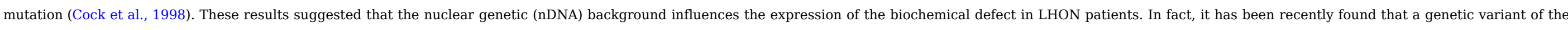
nDNA-encoded mitochondrial enzyme tyrosyl-tRNA synthetase, which decreases OXPHOS capacity, is a nuclear modifier for LHON (Jiang et al., 2016a).

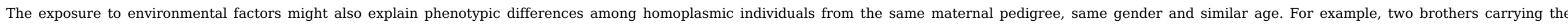

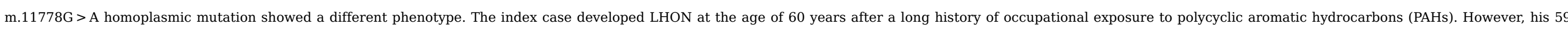

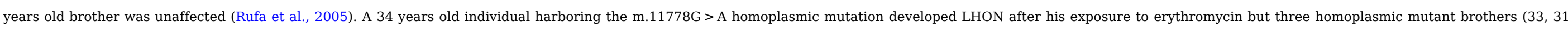

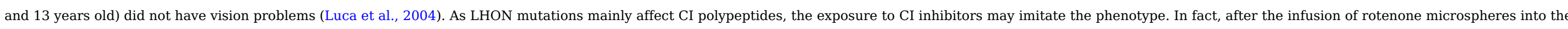

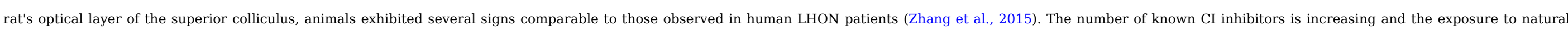

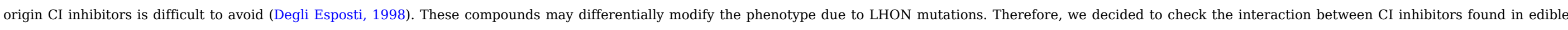
plants, such as capsaicin and rolliniastatin-1 (Fig. 1), and the most frequent LHON mutations. As a control, we used rotenone, a frequently used CI inhibitor.

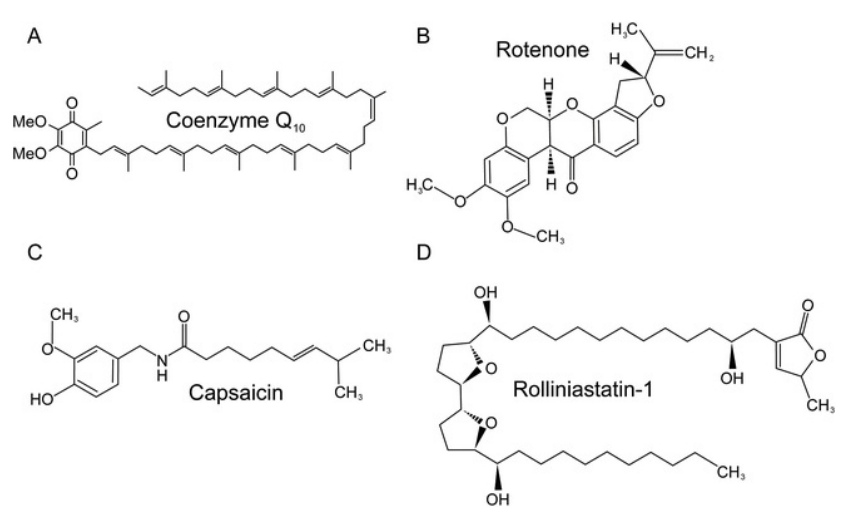

Fig. 1 Respiratory complex I (CI) inhibitors. The structural formula of CI substrate (A) and inhibitors (B-D) are represented.

\section{alt-text: Fig. 1}

\section{Material and methods}

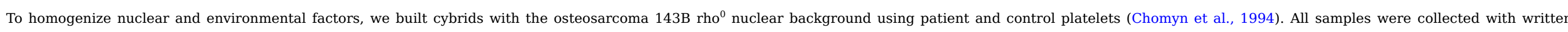

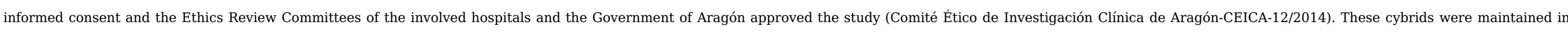

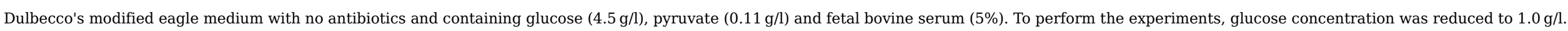

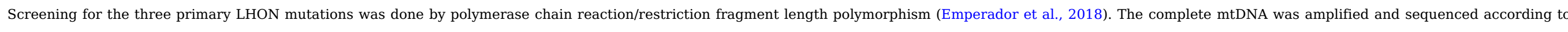

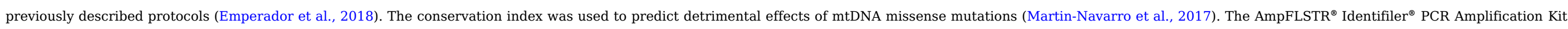
(Life Technologies) was used to determine the cybrids nDNA genetic fingerprints. The molecular cytogenetic analysis was done according to already published procedures (Lopez-Gallardo et al., 2016).

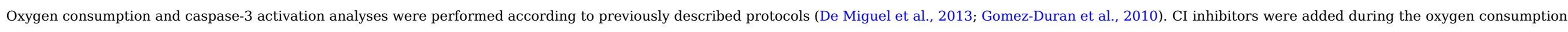
or two days before the caspase-3, studies. 
The three-dimensional structure of the bovine p. MT-ND1 subunit (PDB 5LNK), ortholog of human p. MT-ND1, was obtained with the RasMol 2.6 program (http://www.rasmol.org)

\section{Results}

\subsection{Characterization of cybrid cell lines}

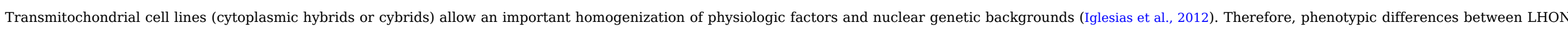
and wild-type cybrids should be mostly due to their mtDNA genotypes.

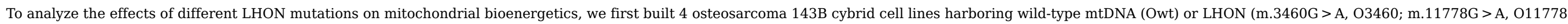

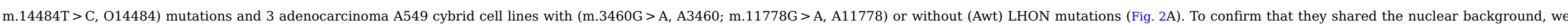

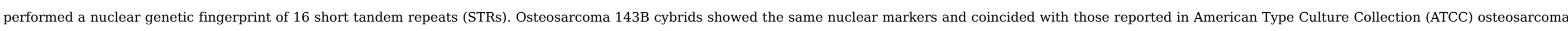

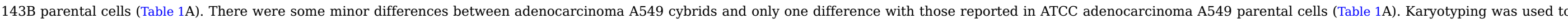

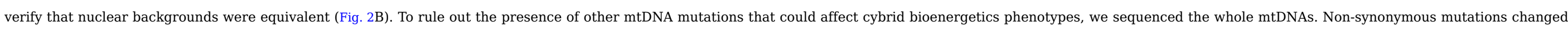
evolutionary poorly conserved amino acids and, probably, with no functional importance (Table 1B).

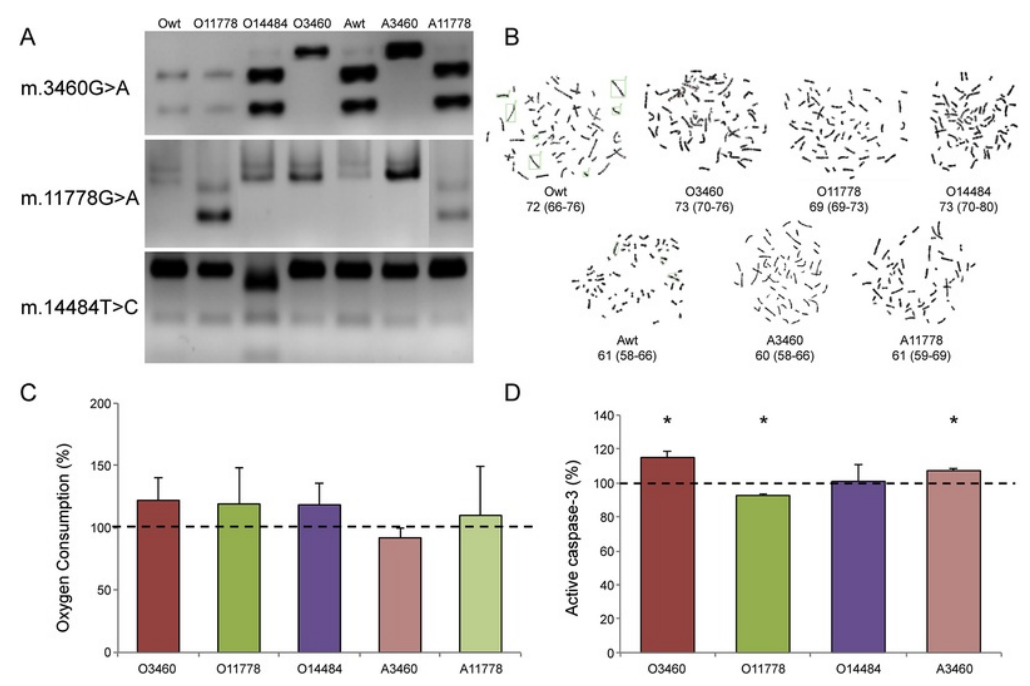

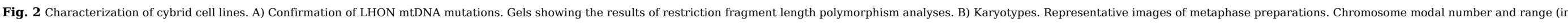

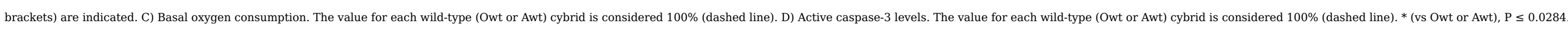
alt-text: Fig. 2

Table 1 Cybrid genetic characterization.

alt-text: Table 1

\begin{tabular}{|c|c|c|c|c|c|c|c|c|c|c|}
\hline \multicolumn{11}{|c|}{ A. Nuclear genetic fingerprint. } \\
\hline $\mathrm{Chr}$ & Marker & $\begin{array}{l}\text { O143B } \\
\text { (ATCC) }\end{array}$ & Owt & O3460 & O11778 & O14484 & $\begin{array}{c}\text { A549 } \\
\text { (ATCC) }\end{array}$ & Awt & A3460 & A11778 \\
\hline 2 & D2S1338 & & 24,25 & 24,25 & 24,25 & 24,25 & & 24,25 & 24,25 & 24,25 \\
\hline 2 & TPOX & 11 & 11 & 11 & 11 & 11 & 8,11 & 8,11 & 8,11 & 8,11 \\
\hline 3 & D.S13.58 & & 15 & 15 & 15 & 15 & & 16 & 16 & 1617 \\
\hline
\end{tabular}




\begin{tabular}{|c|c|c|c|c|c|c|c|c|c|c|}
\hline- & 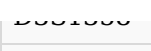 & & 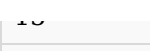 & $\therefore$ & 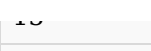 & 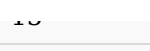 & & $\therefore$ & $\approx$ & $+\infty, \ldots$ \\
\hline 4 & FGA & & 24 & 24 & 24 & 24 & & 23 & 23 & 23 \\
\hline 5 & D5S818 & 13 & 13 & 13 & 13 & 13 & 11 & 11,12 & 11,12 & 11 \\
\hline 5 & CSF1PO & 12 & 12 & 12 & 12 & 12 & 10,12 & 10,12 & 10,12 & 10,12 \\
\hline 7 & D7S820 & 11,12 & 11,12 & 11,12 & 11,12 & 11,12 & 8,11 & 8,11 & 8,11 & 8,11 \\
\hline 8 & D8S1179 & & 11,14 & 11,14 & 11,14 & 11,14 & & 13,14 & 13,14 & 13,14 \\
\hline 11 & TH01 & 6 & 6 & 6 & 6 & 6 & $8,9.3$ & $8,9.3$ & $8,9.3$ & $8,9.3$ \\
\hline 12 & vWA & 18 & 18 & 18 & 18 & 18 & 14 & 14 & 14 & 14 \\
\hline 13 & D13S317 & 12 & 12 & 12 & 12 & 12 & 11 & 11 & 11 & 11 \\
\hline 16 & D16S539 & 10,13 & 10,13 & 10,13 & 10,13 & 10,13 & 11,12 & 11,12 & 11,12 & 11,12 \\
\hline 18 & D18S51 & & 17 & 17 & 17 & 17 & & 14,17 & 14,17 & 14,17 \\
\hline 19 & D19S433 & & 13 & 13 & 13 & 13 & & 13 & 13 & 13 \\
\hline 21 & D21S11 & & $31.2,32.2$ & $31.2,32.2$ & $31.2,32.2$ & $31.2,32.2$ & & 29 & 29 & 29,30 \\
\hline $\mathrm{X}$ & AMEL & $\mathrm{X}$ & $\mathrm{X}$ & $\mathrm{X}$ & $\mathrm{X}$ & $\mathrm{X}$ & $\mathrm{X}, \mathrm{Y}$ & $\mathrm{X}, \mathrm{Y}$ & $\mathrm{X}, \mathrm{Y}$ & $\mathrm{X}, \mathrm{Y}$ \\
\hline
\end{tabular}

Chr, chromosome. ATCC, American type culture collection. Italic numbers indicate differences between A549 cybrids.

\begin{tabular}{|c|c|c|c|c|c|c|c|}
\hline \multicolumn{8}{|c|}{ B. mtDNA sequence } \\
\hline Cybrid & Owt & O3460 & O11778 & O14484 & Awt & A3460 & A11778 \\
\hline GenBank & JN635299 & MH080305 & MH080306 & MH080307 & КT002149 & MH080308 & MH080309 \\
\hline Haplogroup & J1c8a & J1c2e2 & $\mathrm{J} 1 \mathrm{c} 1 \mathrm{~b}$ & J2a2c & U5a1a2b & $\mathrm{T} 2 \mathrm{~b}$ & T2a1b1a1b \\
\hline Pathologic & & 3460 & 11778 & 14484 & & 3460 & 11778 \\
\hline Mutation & & $(M T-N D 1)$ & $(M T-N D 4)$ & $(M T-N D 6)$ & & $(M T-N D 1)$ & $(M T-N D 4)$ \\
\hline $\begin{array}{l}\text { Private } \\
\text { Mutations }\end{array}$ & $\begin{array}{l}185 \\
(M T-D L O O P) \\
\mathbf{3 3 8 7 A} \\
\text { (MT-ND1) } \\
\mathbf{1 4 1 8 9} \\
\text { (MT-ND6) } \\
16241 \\
(\text { MT-DLOOP) }\end{array}$ & $\begin{array}{l}10398 \\
(M T-N D 3) \\
12131 \\
(M T-N D 4) \\
14198 \\
\text { (MT-NDG) }\end{array}$ & $\begin{array}{l}188 \\
(M T-D L O O P) \\
482 \\
(M T-D L O O P) \\
501 \\
(M T-D L O O P) \\
5231 \\
(M T-N D 2) \\
6275 \\
(M T-C O 1)\end{array}$ & $\begin{array}{l}\mathbf{7 2 6 9} \\
\text { (MT-CO1) } \\
11377 \\
(M T-N D 4) \\
14569 \\
(M T-N D 6)\end{array}$ & $\begin{array}{l}15148 \\
(M T-C Y B) \\
15530 \\
(M T-C Y B)\end{array}$ & $\begin{array}{l}12040 \\
(M T-N D 4)\end{array}$ & $\begin{array}{l}1547 \mathrm{Ti} \\
(M T-R N R 1) \\
12121 \\
(M T-N D 4) \\
16051 \\
(M T-D L O O P) \\
16086 \\
(M T-D L O O P) \\
16218 \\
(M T-D L O O P)\end{array}$ \\
\hline
\end{tabular}

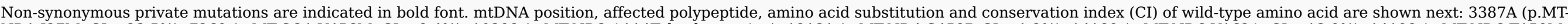

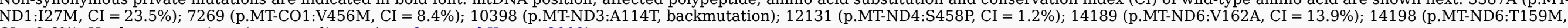
$\mathrm{CI}=2.6 \%$ ). Haplogroup assignation according to (van Oven and Kayser, 2009).

C. Amino acid variation at p.MT.ND1,3 subunits

Cybrid p.MT-ND1

p.MT-ND3 
Cybrid differences in p.MT-ND1,3 amino acids are shown in bold font.

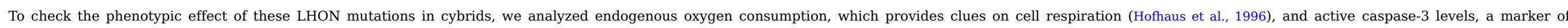

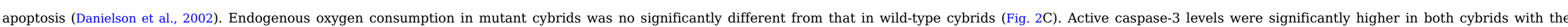
m.3460G > A mutation (O3460 and A3460) and significantly lower in O11778. There was no difference between O14484 and Owt (Fig. 2D).

All these results suggested that, besides LHON mutation, other factors were required for phenotype expression in patients. Below, we explore environmental exposures.

\subsection{Oxygen consumption}

\subsubsection{Rotenone}

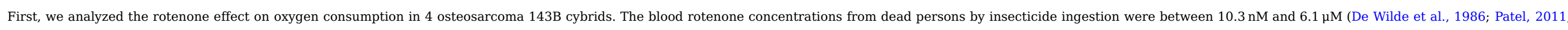

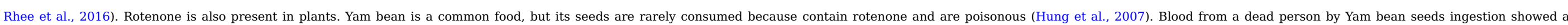
rotenone concentration of $182 \mathrm{nM}$ (Narongchai et al., 2005). After these reports, we selected rotenone concentrations $\leq 6.25 \mathrm{nM}$.

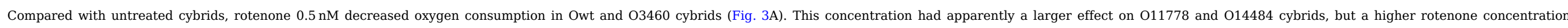

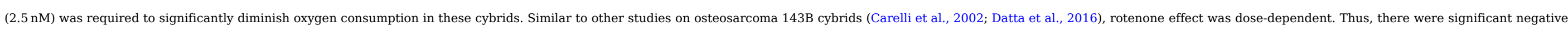

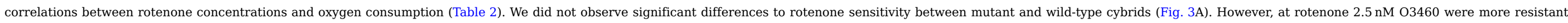
than 011778 cybrids. At rotenone $3.75 \mathrm{nM}$, O3460 were more resistant than 011778 and 014484 cybrids.
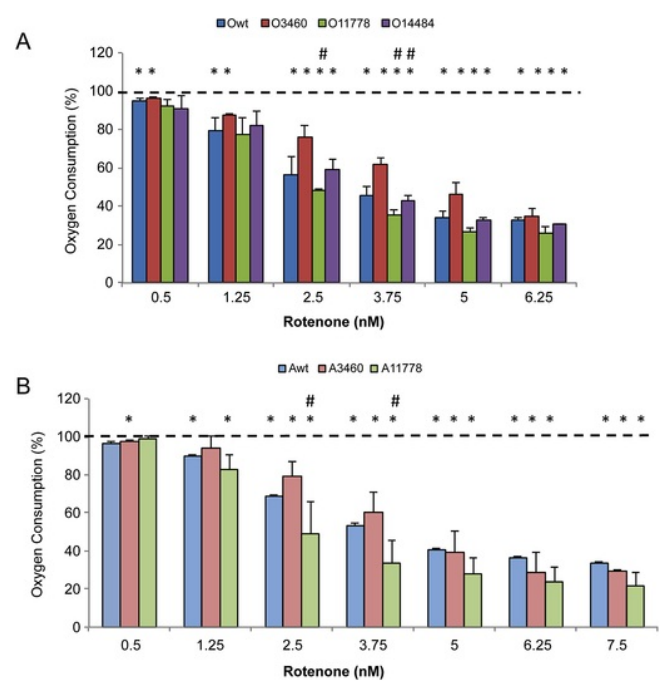

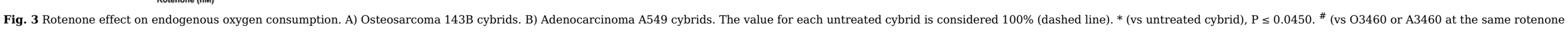
concentration), $\mathrm{P} \leq 0.0496$. 
Table 2 Statistical correlations between xenobiotic concentrations and basal oxygen consumption.

alt-text: Table 2

Cybrid

Rotenone

Capsaicin

Rolliniastatin-1

Osteosarcoma 143B

Owt

$$
\begin{aligned}
& y=-11.0 x+92.8 \\
& R^{2}=0.9215
\end{aligned}
$$

O3460

$\mathrm{y}=-10.8 \mathrm{x}+101.7$

$$
\mathrm{R}^{2}=0.9982
$$

O11778

$$
\begin{aligned}
& \mathrm{y}=-11.9 \mathrm{x}+89.1 \\
& \mathrm{R}^{2}=0.8867
\end{aligned}
$$

$\mathrm{y}=-2.0 \mathrm{x}+115.0$

Capsaicin

\begin{tabular}{|c|c|c|c|}
\hline \multirow[t]{2}{*}{ Awt } & $y=-11.2 x+100.2$ & $y=-1.9 x+114.1$ & $y=-0.07 x+105.6$ \\
\hline & $\mathrm{R}^{2}=0.9674$ & $\mathrm{R}^{2}=0.9742$ & $\mathrm{R}^{2}=0.9876$ \\
\hline \multirow[t]{2}{*}{ A3460 } & $y=-13.0 x+108.2$ & $y=-2.2 x+85.8$ & $y=-0.11 x+101.7$ \\
\hline & $\mathrm{R}^{2}=0.9870$ & $\mathrm{R}^{2}=0.9282$ & $\mathrm{R}^{2}=0.9990$ \\
\hline \multirow[t]{2}{*}{ A11778 } & $y=-13.3 x+95.4$ & $y=-1.5 x+101.7$ & $y=-0.11 x+113.9$ \\
\hline & $\mathrm{R}^{2}=0.8861$ & $\mathrm{R}^{2}=0.9803$ & $\mathrm{R}^{2}=0.9819$ \\
\hline
\end{tabular}

\begin{tabular}{|l|l|}
\hline O14484 & $\mathrm{y}=-11.2 \mathrm{x}+92.4$ \\
\hline $\mathrm{R}^{2}=0.9404$
\end{tabular}

$$
\begin{aligned}
& \mathrm{y}=-2.0 \mathrm{x}+115.0 \\
& \mathrm{R}^{2}=0.9964 \\
& \mathrm{y}=-2.0 \mathrm{x}+91.9 \\
& \mathrm{R}^{2}=0.9535 \\
& \mathrm{y}=-1.6 \mathrm{x}+103.7 \\
& \mathrm{R}^{2}=0.9983 \\
& \mathrm{y}=-1.9 \mathrm{x}+105.8 \\
& \mathrm{R}^{2}=0.9990
\end{aligned}
$$

$$
\begin{aligned}
& y=-0.12 x+114.0 \\
& R^{2}=0.9582 \\
& y=-0.15 x+109.3 \\
& R^{2}=0.9582 \\
& y=-0.08 x+109.1 \\
& R^{2}=0.9802
\end{aligned}
$$

Adenocarcinoma A549

All correlations were statistically significant $(\mathrm{P}<0.0001)$.

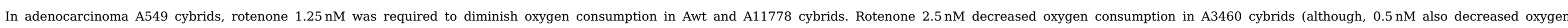

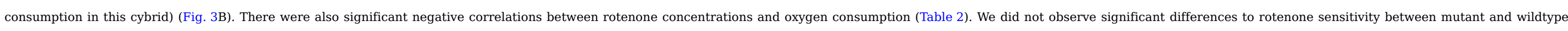

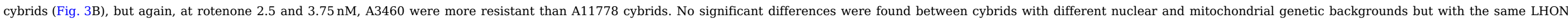
mutation and drug concentration. These results suggested that $\mathrm{m} .3460 \mathrm{G}>\mathrm{A}$ mutation modified the rotenone phenotypic effect but other nDNA and mtDNA factors had less effect.

\subsubsection{Capsaicin}

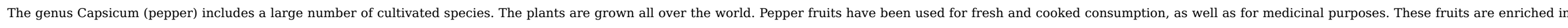

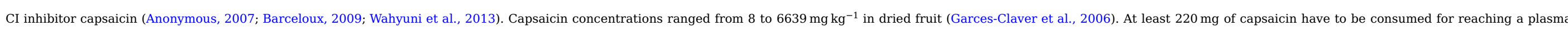

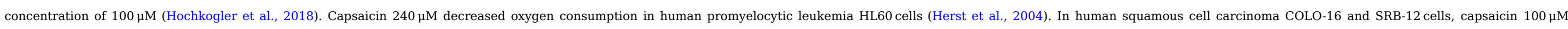

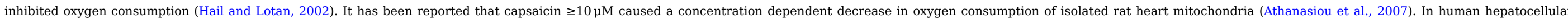

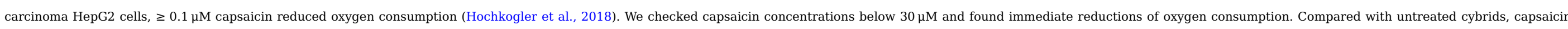

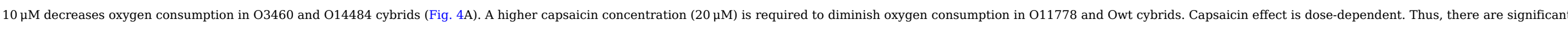

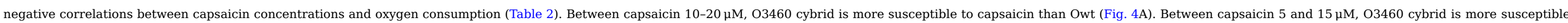


A

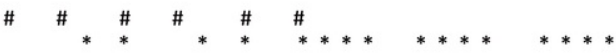

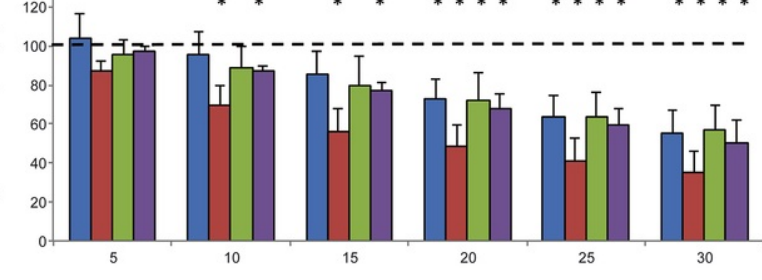

Capsaicin ( $\mu$ M)

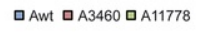

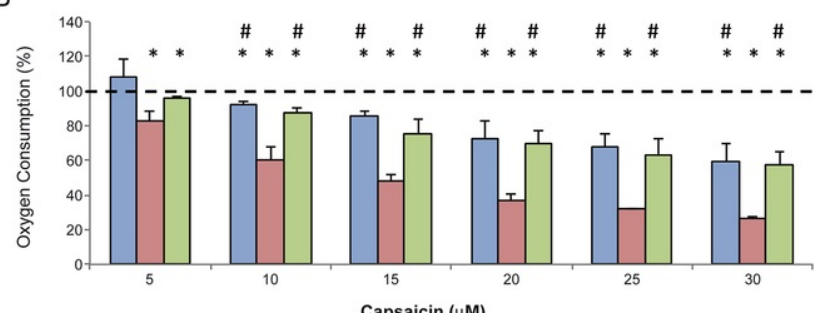

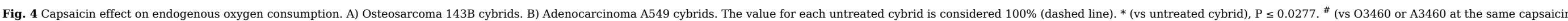
concentration), $\mathrm{P} \leq 0.0458$.

\section{alt-text: Fig. 4}

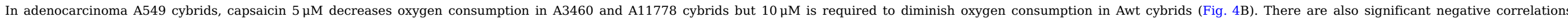

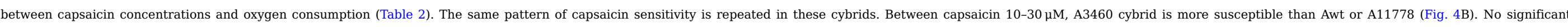

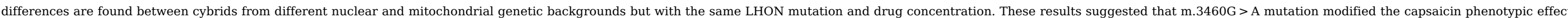
but other nDNA and mtDNA factors had less effect.

\subsubsection{Rolliniastatin-1}

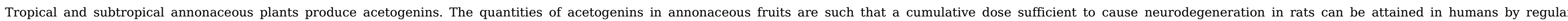

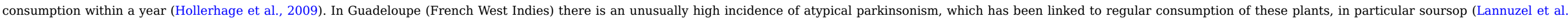

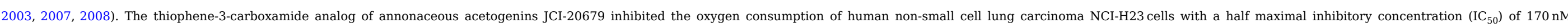

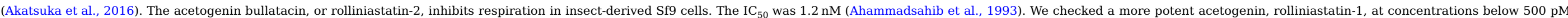
in osteosarcoma 143B and adenocarcinoma A549 cybrids.

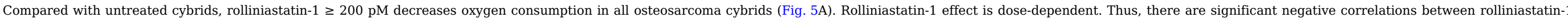

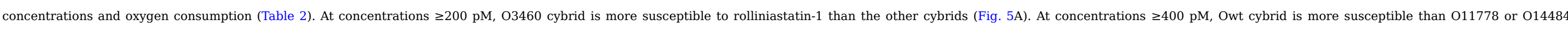
cybrids (Fig. 5A). 


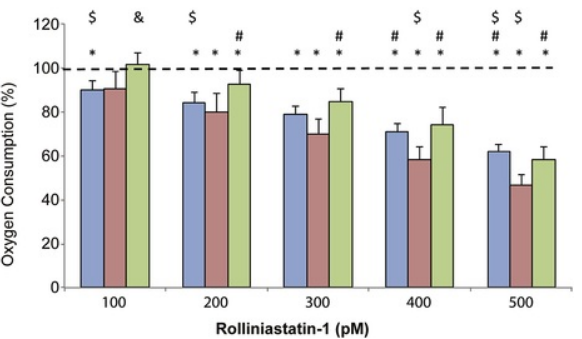

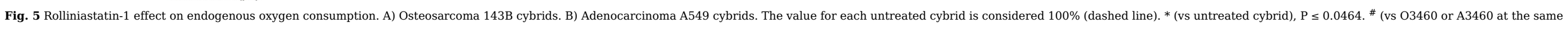
rolliniastatin-1 concentration), $\mathrm{P} \leq 0.0448$. ${ }^{\&}$ (vs Owt or Awt at the same rolliniastatin-1 concentration), $\mathrm{P} \leq 0.0448$. ${ }^{\$}$ (vs Owt or $\mathrm{O} 3460$ at the same rolliniastatin-1 concentration), $\mathrm{P} \leq 0.0348$.

\section{alt-text: Fig. 5}

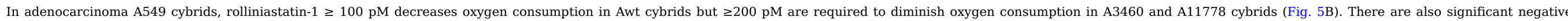

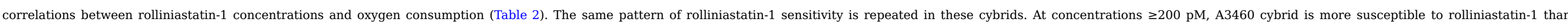

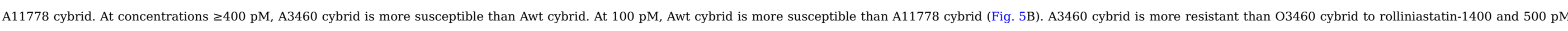

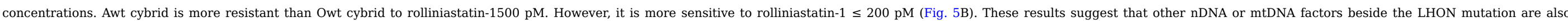
involved in the rolliniastatin-1 susceptibility.

\subsection{Apoptosis}

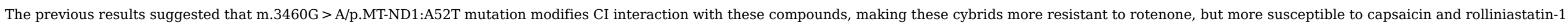

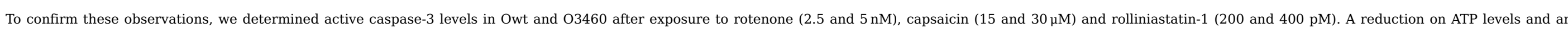

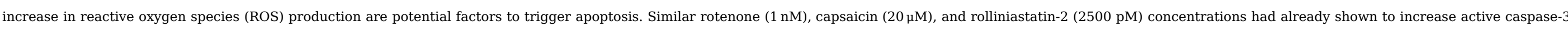

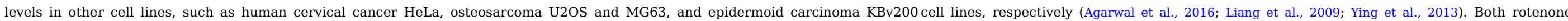

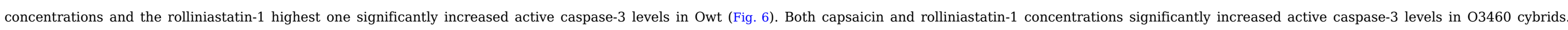

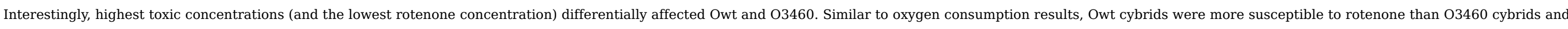

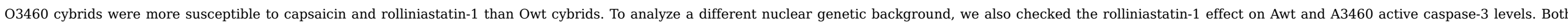

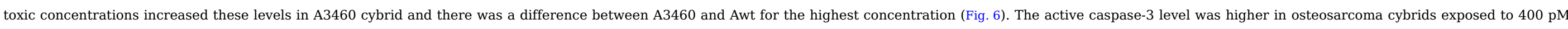
rolliniastatin-1 than in adecarcinoma ones. Therefore, they were more susceptible to this drug, confirming the result obtained for endogenous oxygen consumption. 


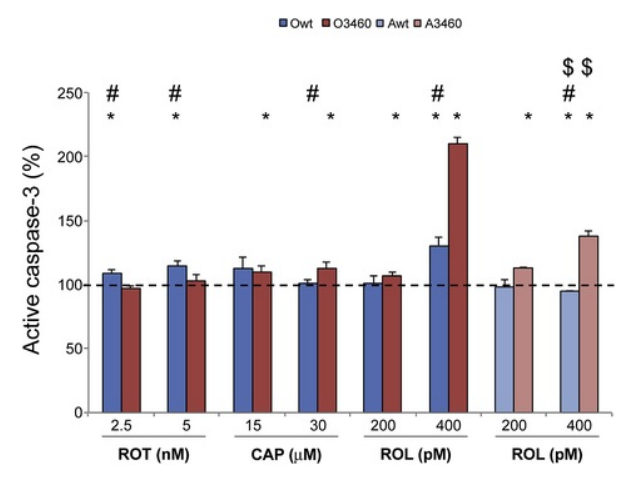

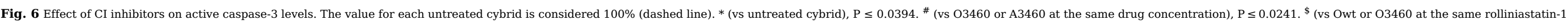
concentration), $\mathrm{P} \leq 0.0006$.

alt-text: Fig. 6

\section{Discussion}

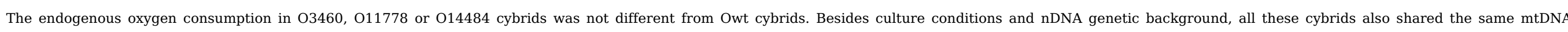

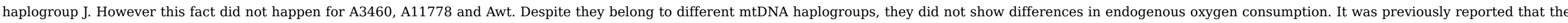

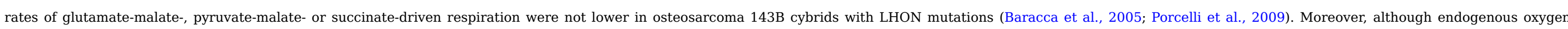

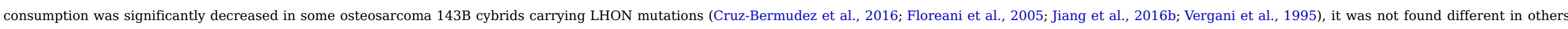

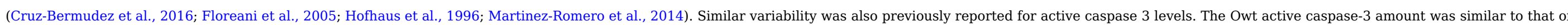

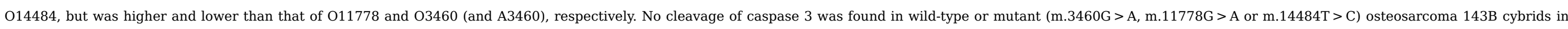
another study (Zanna et al., 2003). Moreover, no differences in active caspase-3 levels were found between wild-type and m.11778G > A osteosarcoma 143B cybrids (Danielson et al., 2002).

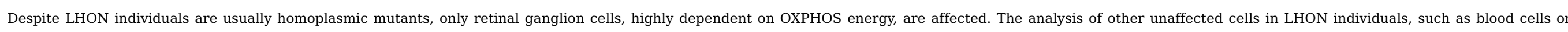

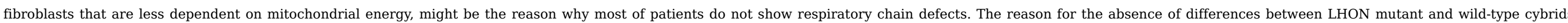

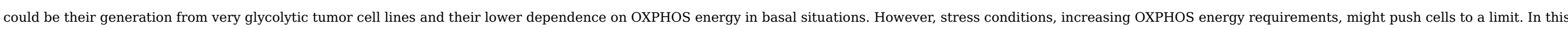

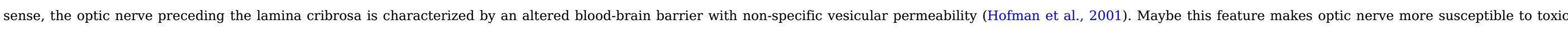

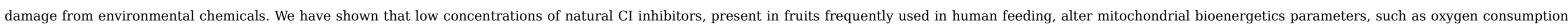

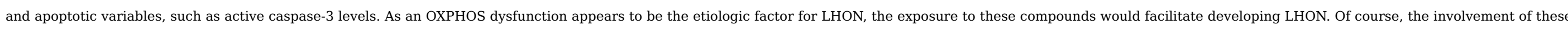
fruits as LHON triggers would require more investigation (see https://scienceofparkinsons.com/2017/12/16/paq/\#more-48745).

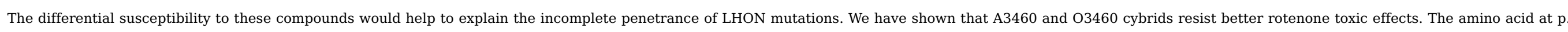

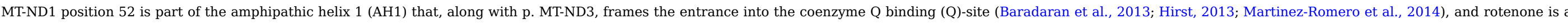

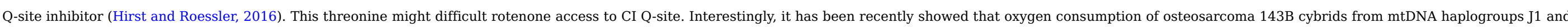

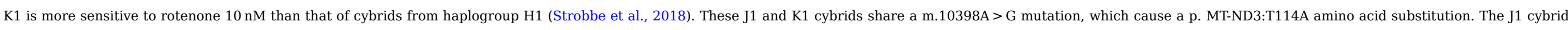

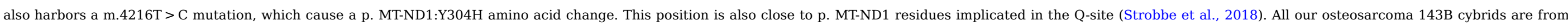

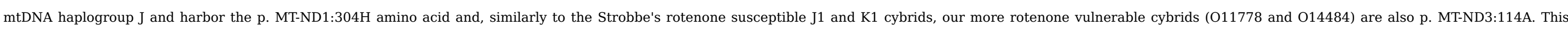

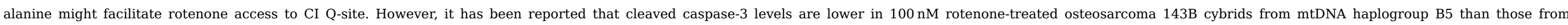

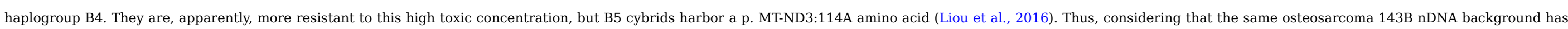
been used in these three publications, other factors must be also involved in rotenone susceptibility. 


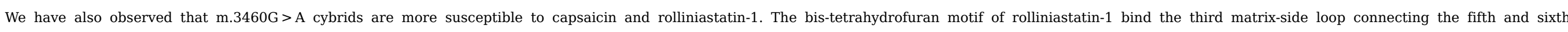

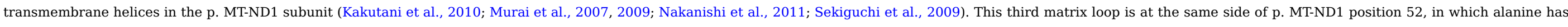

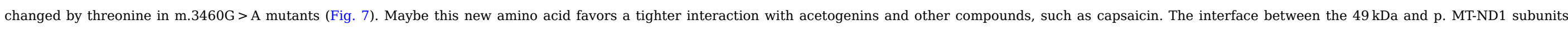
forms the inhibitor/Q-site, although it is considered that different CI inhibitors act on the same Q-site with different but partially overlapping sites (Okun et al., 1999; Sinha et al., 2015).

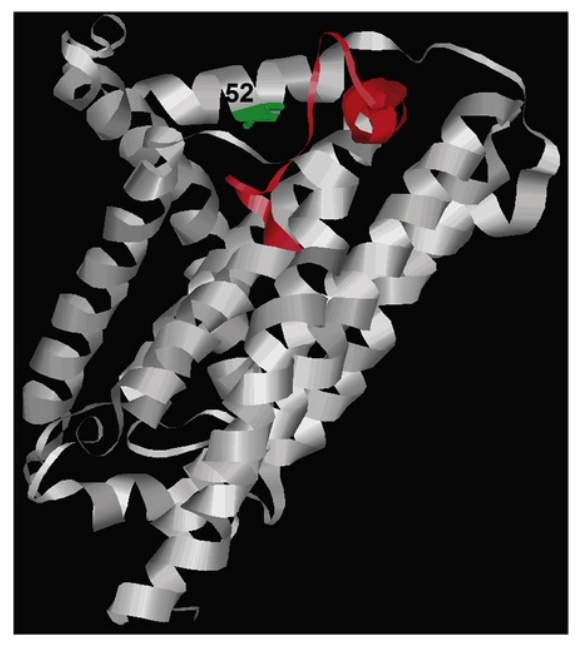

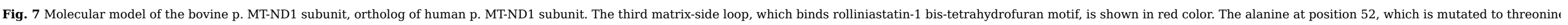
in $\mathrm{m} .3460 \mathrm{G}>\mathrm{A}$, is represented in green color. (For interpretation of the references to color in this figure legend, the reader is referred to the Web version of this article.)

\section{alt-text: Fig. 7}

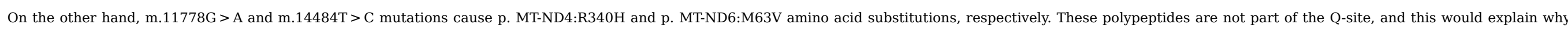

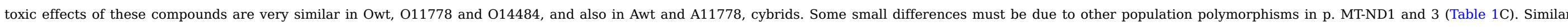

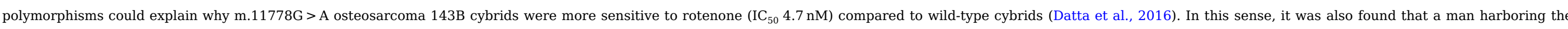

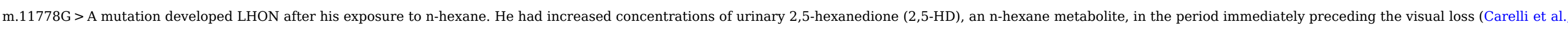

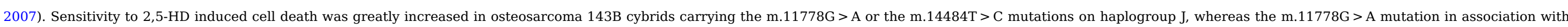
haplogroups U and H significantly improved cell survival. Thus, mtDNA genetic background can modify the interaction with environmental factors (Ghelli et al., 2009).

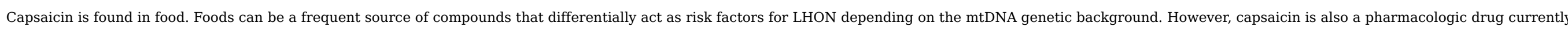

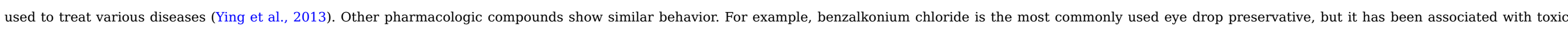

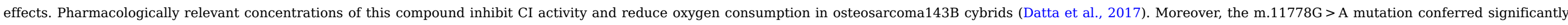
increased sensitivity to benzalkonium chloride (Datta et al., 2017).

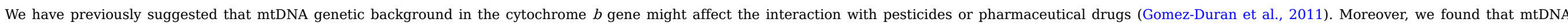

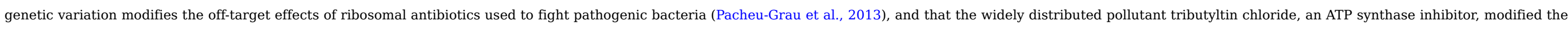

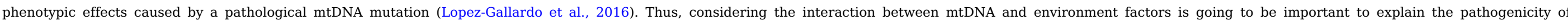
mitochondrial disease due to mtDNA mutations.

\section{Conflicts of interest}

The authors declare no conflict of interest. 


\section{Acknowledgements}

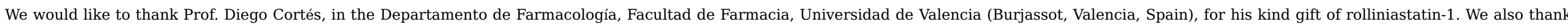

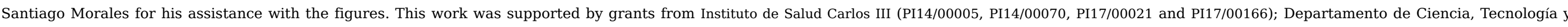

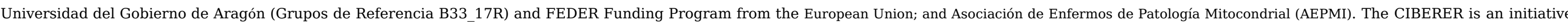

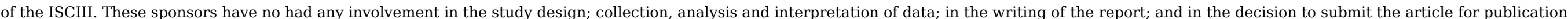

\section{Transparency document}

Transparency document related to this article can be found online at https://doi:10.1016/j.fct.2018.07.014.

\section{References}

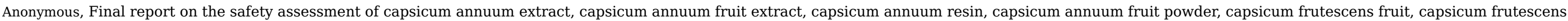
fruit extract, capsicum frutescens resin, and capsaicin, Int. J. Toxicol. 26 (Suppl. 1), 2007, 3-106.

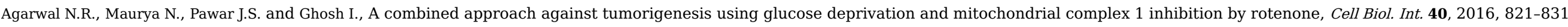

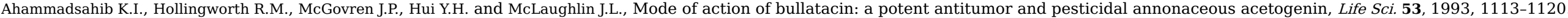

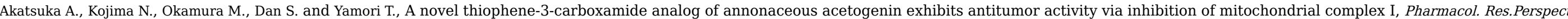
4, 2016, e00246.

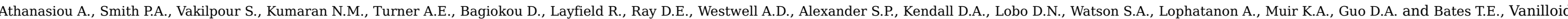
receptor agonists and antagonists are mitochondrial inhibitors: how vanilloids cause non-vanilloid receptor mediated cell death, Biochem. Biophys. Res. Commun. 354, $2007,50-55$.

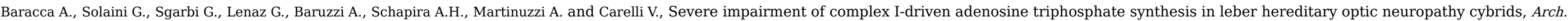
Neurol. 62, 2005, 730-736.

Baradaran R., Berrisford J.M., Minhas G.S. and Sazanov L.A., Crystal structure of the entire respiratory complex I, Nature 494, 2013, 443-448.

Barceloux D.G., Pepper and capsaicin (capsicum and piper species), Dis Mon 55, 2009, 380-390.

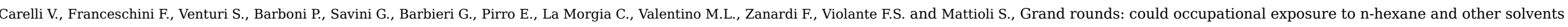
precipitate visual failure in leber hereditary optic neuropathy?, Environ. Health Perspect. 115, 2007, 113-115.

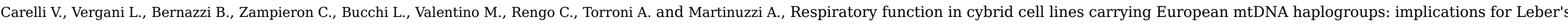
hereditary optic neuropathy, Biochim. Biophys. Acta 1588, 2002, 7-14.

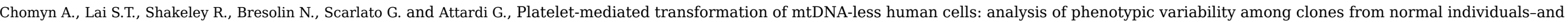
complementation behavior of the tRNALys mutation causing myoclonic epilepsy and ragged red fibers, Am. J. Hum. Genet. 54, 1994, 966-974.

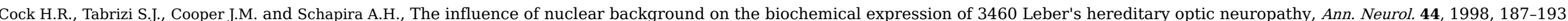

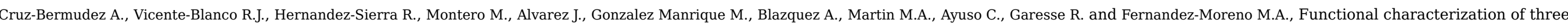
concomitant MtDNA LHON mutations shows No synergistic effect on mitochondrial activity, PLoS One 11, 2016, e0146816.

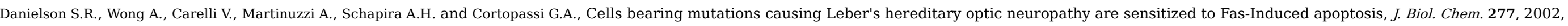
5810-5815. 


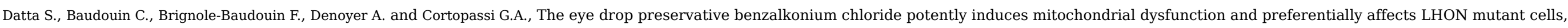
Invest. Ophthalmol. Vis. Sci. 58, 2017, 2406-2412.

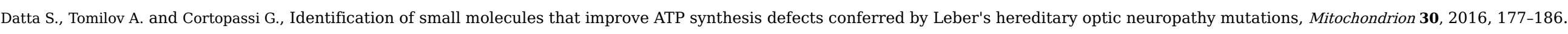

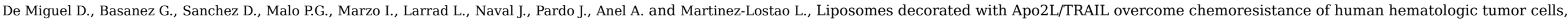
Mol. Pharm. 10, 2013, 893-904.

De Wilde A.R., Heyndrickx A. and Carton D., A case of fatal rotenone poisoning in a child, J. Forensic Sci. 31, 1986, 1492-1498

Degli Esposti M., Inhibitors of NADH-ubiquinone reductase: an overview, Biochim. Biophys. Acta 1364, 1998, 222-235.

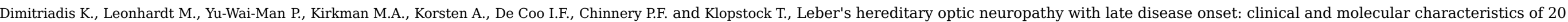
patients, Orphanet J. Rare Dis. 9, 2014, 158.

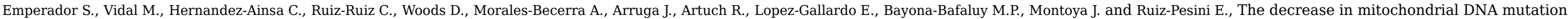
load parallels visual recovery in a leber hereditary optic neuropathy patient, Front. Neurosci. 12, 2018, 61.

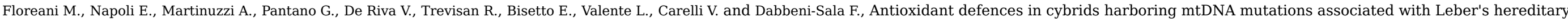
optic neuropathy, FEBS J. 272, 2005, 1124-1135.

Franks W.A. and Sanders M.D., Leber's hereditary optic neuropathy in women, Eye 4 (Pt 3), 1990, 482-485.

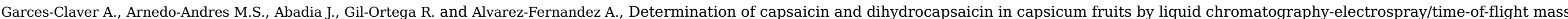
spectrometry, J. Agric. Food Chem. 54, 2006, 9303-9311.

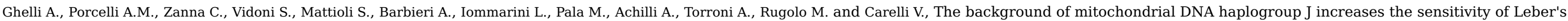
hereditary optic neuropathy cells to 2,5-hexanedione toxicity, PLoS One 4, 2009, e7922.

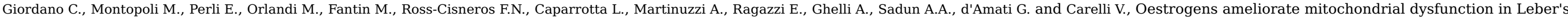
hereditary optic neuropathy, Brain 134, 2011, 220-234.

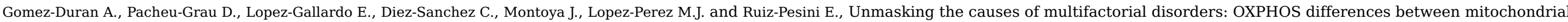
haplogroups, Hum. Mol. Genet. 19, 2010, 3343-3353.

Gomez-Duran A., Pacheu-Grau D., Lopez-Perez M.J., Montoya J. and Ruiz-Pesini E., Mitochondrial pharma-Q-genomics: targeting the OXPHOS cytochrome b, Drug Discov. Today 16, 2011, 176-180.

Hail N., Jr. and Lotan R., Examining the role of mitochondrial respiration in vanilloid-induced apoptosis, J. Natl. Cancer Inst. 94, 2002, 1281-1292.

Herst P.M., Tan A.S., Scarlett D.J. and Berridge M.V., Cell surface oxygen consumption by mitochondrial gene knockout cells, Biochim. Biophys. Acta 1656, $2004,79-87$.

Hirst J., Mitochondrial complex I, Annu. Rev. Biochem. 82, 2013, 551-575.

Hirst J. and Roessler M.M., Energy conversion, redox catalysis and generation of reactive oxygen species by respiratory complex I, Biochim. Biophys. Acta 1857, 2016, 872-883.

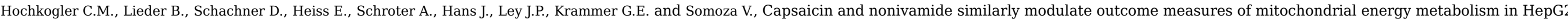
and 3T3-L1 cells, Food Funct 9, 2018, 1123-1132.

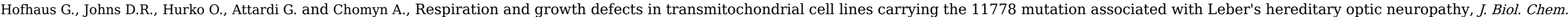


271, 1996, 13155-13161.

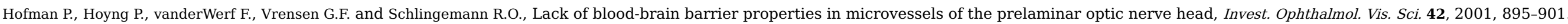

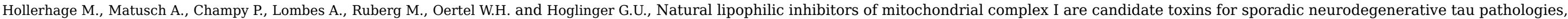
Exp. Neurol. 220, 2009, 133-142.

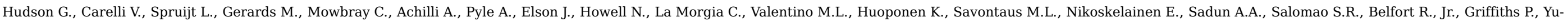

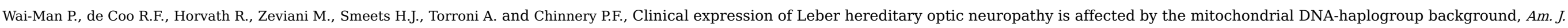
Hum. Genet. 81, 2007, 228-233.

Hung Y.M., Hung S.Y., Olson K.R., Chou K.J., Lin S.L., Chung H.M., Tung C.N. and Chang J.C., Yam bean seed poisoning mimicking cyanide intoxication, Intern. Med. J. 37, 2007, 130-132.

Iglesias E., Llobet L., Pacheu-Grau D., Gomez-Duran A. and Ruiz-Pesini E., Cybrids for mitochondrial DNA pharmacogenomics, Drug Dev. Res. 73, 2012, 453-460.

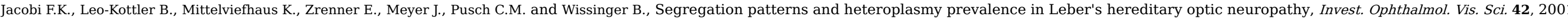
$1208-1214$.

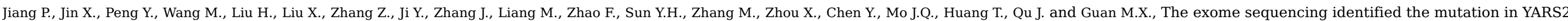

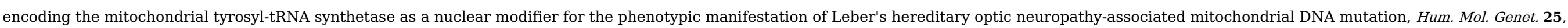
2016a, 584-596

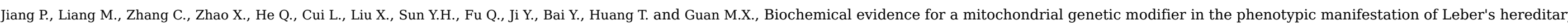
optic neuropathy-associated mitochondrial DNA mutation, Hum. Mol. Genet. 25, 2016b, 3613-3625.

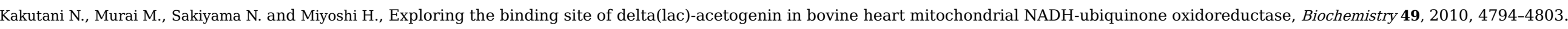

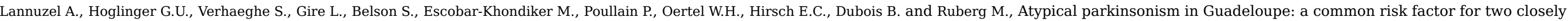
related phenotypes?, Brain 130, 2007, 816-827.

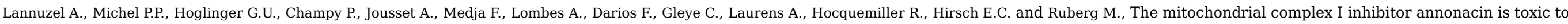
mesencephalic dopaminergic neurons by impairment of energy metabolism, Neuroscience 121, 2003, 287-296.

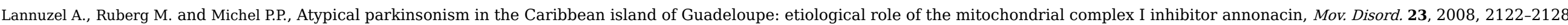

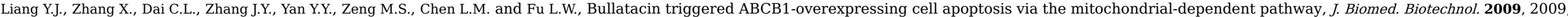
867123.

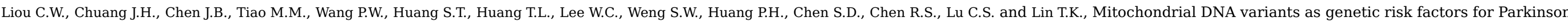
disease, Eur. J. Neurol. 23, 2016, 1289-1300.

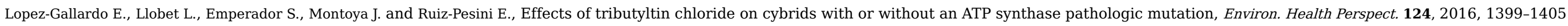

Luca C.C., Lam B.L. and Moraes C.T., Erythromycin as a potential precipitating agent in the onset of Leber's hereditary optic neuropathy, Mitochondrion 4, $2004,31-36$.

Maresca A., Caporali L., Strobbe D., Zanna C., Malavolta D., La Morgia C., Valentino M.L. and Carelli V., Genetic basis of mitochondrial optic neuropathies, Curr. Mol. Med. 14, 2014, 985-992.

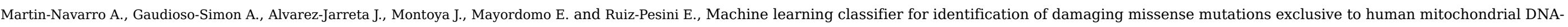
encoded polypeptides, BMC Bioinf. 18, 2017, 158 


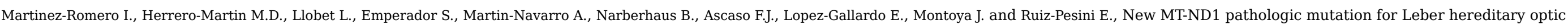
neuropathy, Clin. Exp. Ophthalmol. 42, 2014, 856-864.

Murai M., Ishihara A., Nishioka T., Yagi T. and Miyoshi H., The ND1 subunit constructs the inhibitor binding domain in bovine heart mitochondrial complex I, Biochemistry 46, 2007, 6409-6416.

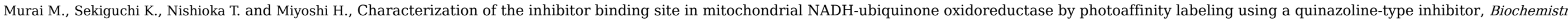
48, 2009, 688-698.

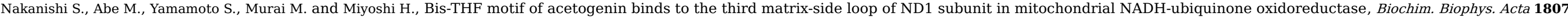
2011, 1170-1176.

Narongchai P., Narongchai S. and Thampituk S., The first fatal case of yam bean and rotenone toxicity in Thailand, J. Med. Assoc. Thai. 88, 2005, 984-987.

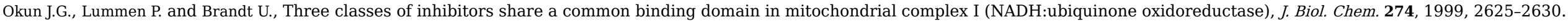

Pacheu-Grau D., Gomez-Duran A., Iglesias E., Lopez-Gallardo E., Montoya J. and Ruiz-Pesini E., Mitochondrial antibiograms in personalized medicine, Hum. Mol. Genet. 22, 2013, 1132-1139.

Patel F., Pesticidal suicide: adult fatal rotenone poisoning, J Forensic Leg Med 18, 2011, 340-342.

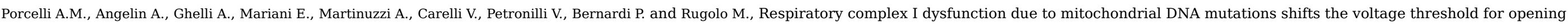
of the permeability transition pore toward resting levels, J. Biol. Chem. 284, 2009, 2045-2052.

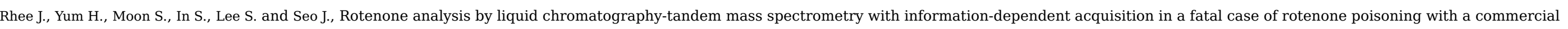
organic insecticide being sold in korea, J. Anal. Toxicol. 40, 2016, 460-465.

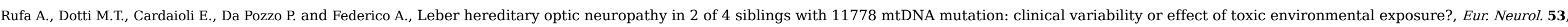
2005, 32-34.

Sekiguchi K., Murai M. and Miyoshi H., Exploring the binding site of acetogenin in the ND1 subunit of bovine mitochondrial complex I, Biochim. Biophys. Acta 1787, 2009, 1106-1111.

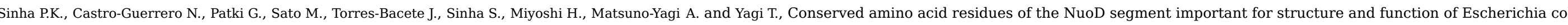
NDH-1 (complex I), Biochemistry 54, 2015, 753-764.

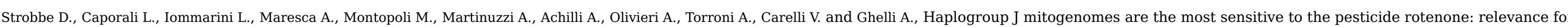
human diseases, Neurobiol. Dis. 114, 2018, 129-139.

van Oven M. and Kayser M., Updated comprehensive phylogenetic tree of global human mitochondrial DNA variation, Hum. Mutat. 30, 2009, E386-E394.

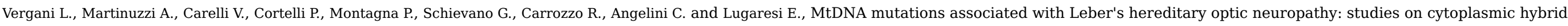
(cybrid) cells, Biochem. Biophys. Res. Commun. 210, 1995, 880-888.

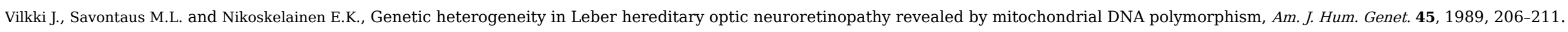

Wahyuni Y., Ballester A.R., Sudarmonowati E., Bino R.J. and Bovy A.G., Secondary metabolites of Capsicum species and their importance in the human diet, J. Nat. Prod. 76, 2013, 783-793.

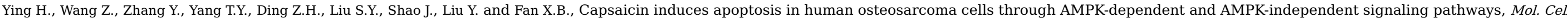
Biochem. 384, 2013, 229-237.

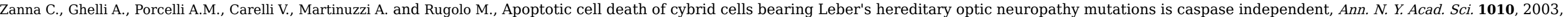


213-217.

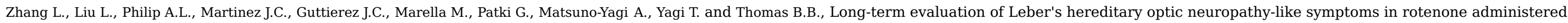
rats, Neurosci. Lett. 585, 2015, 171-176.

\section{Transparency document}

Multimedia Component 1

coi_disclosure CHA

alt-text: coi_disclosure CHA

\section{Highlights}

- Some mitochondrial DNA (mtDNA) mutations cause Leber hereditary optic neuropathy (LHON).

- Some oxidative phosphorylation system inhibitors cause mitochondrial optic neuropathies.

- Food derived xenobiotics modify the penetrance of LHON mtDNA mutations.

- The m.3460G > A mutation increases rotenone resistance but capsaicin and rolliniastatin-1 susceptibility.

- Gene-environment interactions must be considered to explain mitochondrial disease pathogenicity.

\section{Queries and Answers}

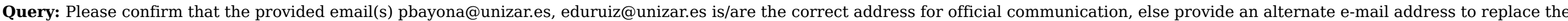
existing one, because private e-mail addresses should not be used in articles as the address for communication.

Answer: pbayonar@unizar.es and eduruiz@unizar.es are the correct addresses for official communication. None of the provided e-emails are private addresses.

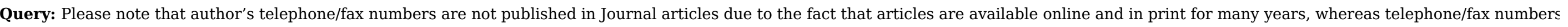
are changeable and therefore not reliable in the long term.

Answer: OK

Query: Please check whether the order of designated corresponding authors are correct, and amend if necessary.

Answer: OK

Query: Could you please provide the grant number for European Union; and Asociación de Enfermos de Patología Mitocondrial, if any?

Answer: There are no grant numbers for these Organisms.

Query: Please check the layout of Table 1, and correct if necessary.

Answer: OK

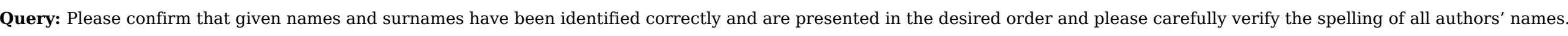

Answer: OK 
Answer: Our article is for inclusion in a regular issue of the journal. 